\title{
Design of Purebred Dog Recommendation System Using MCDM Approach
}

Phie Chyan*

Department of Informatics, Atma Jaya University of Makassar, Makassar, 90224, Indonesia

\begin{tabular}{l} 
A R T I C L E I N F O \\
\hline Article history: \\
Received: 15 May, 2020 \\
Accepted: 18 August, 2020 \\
Online: 09 September, 2020 \\
\hline Keywords: \\
Dog Breed \\
Dog Adopter \\
Purebred Dog \\
Decision Support System \\
MCDM Approach \\
\hline
\end{tabular}

\begin{tabular}{l} 
A B S T R A C T \\
\hline The dog is one of the first animals domesticated by human, and for thousands of years, it \\
has been artificially bred into hundreds of types in order to provide certain traits that \\
humans want. Nowadays, the selection of dogs by potential adopters has become a problem \\
due to the availability of different type of breeds with their physical and mental \\
characteristics. This study aims to design a decision support system through an analytical \\
model that uses variety of data on the characteristics of purebred dogs obtained from \\
different sources. Data from official sources is obtained from international purebred dog \\
organizations that set the standards for each breed type, while data from unofficial sources \\
is obtained from the dog lovers community, experts, and kennel owners. The result of the \\
study provides appropriate recommendations to potential adopters in selecting a breed that \\
suits their preferences and needs.
\end{tabular}

\section{Introduction}

This paper is an extension of work originally presented in 2018 International Seminar on Research of Information Technology and Intelligent Systems (ISRITI) [1]. According to the history recorded since 15 thousand years ago, Dogs were one of the first animals domesticated by humans in East Asia and then spread throughout the world and became involved in the long history of human culture and civilization. Through artificial selection, humans breed dogs into a new kind of breed by mating two species of dogs that have certain characteristics and traits to accentuate the desired combination of their traits [2]. Nowadays dogs have developed into hundreds of breeds with a total of 344 breeds officially recognized by FCI. Among the many types of breeds, the dog has divided into several breed groups with similar physical and psychological characteristics. Each breed group has certain characteristics that can support the role of the dog in social aspects with human's society [3]. Some types of dogs are used by humans to help in herding cattle, others are used as guard dogs to help law enforcement work as sniffer dogs.

The term dog refers to the dog domesticated by Canis lupus familiaris. Dogs were classified as Canis familiaris by Linnaeus in 1758 but in 1993 by the Smithsonian Institute and the American Mammal Experts Association, dogs were designated as subspecies of the gray wolf Canis lupus. Dogs are social animals, but the dog's personality and behavior can vary depending on the breed. In addition to genetic traits, the dog's personality and behavior also

${ }^{*}$ Corresponding Author: Phie Chyan, Email: Phie_chyan@lecturer.uajm.ac.id depend on the caring environment and treatment received from the dog's owner and people who interact closely with the dog.

Dogs as social animal have a vast variety of personality and are among the most high intelligence animals according to scientific research and field evidence. The level of intelligence of the dog depends on the race and each dog individually. According to Coren (2006), a dog psychologist who conducts dog intelligence studies on more than 100 breeds, the standard of intelligence of a dog is how quickly the dog can follow a new command given and how obedient the dog is to the commands it has learned [4]. Another proof is according to research at the University of Lincoln published, dogs have human-like intelligence who can psychologically recognize emotions and other human psychological states, and this ability is only demonstrated by dogs in interacting with humans, this shows the cognitive social abilities of a dog are more advanced than any other animal that is genetically closer to humans, and this ability is a genetic shift that distinguishes dogs from their wolf ancestors [5].

Based on the physical attributes dogs vary greatly in size, appearance, and behavior compared to other pets. Several of the important physical attribute of dogs highlighted as follows : Dogs have strong muscles, sturdy ankle bones, cardiovascular systems that support physical endurance and running speed, and teeth to catch and tear their prey. Dogs technically walk on tiptoes with toes. In term of sense of sight, research shows dogs can see several colors, although not as much and bright as can be seen by humans with a brightness level of approximately half of the accuracy of 
human vision [6]. In sense of hearing aspect dogs can hear lowfrequency sound range from 16 to $20 \mathrm{~Hz}$ (humans only hear frequencies of 20-70 Hz), and high-frequency sounds range from $70 \mathrm{kHz}$ to $100 \mathrm{kHz}$ (humans only hear frequencies $13-20 \mathrm{kHz}$ ) and from the sense of smell Dogs have nearly 220 million olfactory cells that are sensitive to odors. According to research conducted by Michell (1999) on the age of various breeds of dogs, dog life expectancy depends on the breed with a median value between 1013 years [7].

Breed standard is a set of guidelines which is used to ensure that the dog produced by a breeder or breeding facility conforms to the specifics of the standardized breed. Breed standards are devised by breed associations not by individuals and are written to reflect the use or purpose of the species and breed of the dog [8]. Breed standards help define the ideal dog of a breed and provide goals for breeders in improving stock. In essence, a breed standard is a blueprint for an dog fit for the function it was bred - i.e. herding, tracking etc. For that purposes purebred dogs have a nonprofit organization/federation that aims to standardize and register purebred dogs that will improve the quality of breeding and use of purebred dogs whose health and physical characteristics meet the standards. Federation Cynologique Internationale(FCI) is the parent organization that oversees almost all organizations/kennels from various countries except the USA, Canada, and the United Kingdom. The American Kennel Club (AKC) is a registry of purebred dog pedigrees in the United States that also has an international reputation. At present, FCI and AKC are the world's largest breed of dog registration institutions that establish the standardization and grouping of purebred dogs, until now various dog organizations in the world use the standards issued by FCI and $\mathrm{AKC}$ as guidelines in identifying dog breeds.

Humans are faced with various problems in the selection of dog breed, there are so many breeds available with each of their feature and characteristics and should all of those have aligned with human needs as prospective adopters who also have their respective interests [9]. Selection of the breed that is not compatible can bring problems for both the dog itself and the adopter, a dog may not be well taken care of and even end up in the dog shelter only because of incompatibility with the expectations of the adopter, while the time and cost had wasted for the adopter. More criteria need to be considered by prospective dog adopters apart from physical form characteristics like body size and structure such as the average intelligence level of a breed, ease of care, innate traits, and more. So many criteria that must be considered by prospective adopters in choosing breed types makes decision making a complicated task because of the difficulty in analyzing the influence, interests, and relation between one criteria with another and coupled with many types of breeds available that make this problem requires MultiCriteria Decision Making (MCDM) approach to handle it. The purpose of this study is to design a Decision Support System (DSS) that can provide analytical recommendations using MCDM approach for the problem of selecting a breed of dog that suit the needs and preferences of the potential adopters.

\section{Methodology}

\subsection{Data Sources}

Primary data for this study came from FCI and AKC's online knowledge base sources that contain information on officially recognized breeds, groupings, and their physical and psychological characteristics. Secondary data sources came from interviews conducted with the dog lovers community, dog experts, veterinarians, and kennel owners to find out the experience of the respondents according to their fields and expertise regarding how to choose, care, and handle the breed of dog to be adopted including obstacles encountered in dealing with various problems involving purebred dogs. Data collected from primary and secondary sources were then processed to become a knowledge base of the designed system.

\subsection{Analytic Model}

To support the developed system model, an MCDM based approach is needed in analyzing data sources to produce an appropriate analytical model [10]. The basic principles of developing an analytical model based on MCDM approach are :

- Decomposition : After defining the problem regarding the selection of dog breeds, then the main problem-solving process was carried out into more specific elements to support structure of the main problem, this process must be carried out until the most specific sub-elements are obtained and formed the hierarchical model.

- Comparative Judgment : Conduct an assessment of the elements relative importance in decision making for the selection of dog breeds at a certain level related to elements at a higher level. The assessment results would be presented in the form of pairwise comparison to get the relative weights among the elements.

- Synthesis of Priority : Each pairwise comparison matrix needs to compute to get local priority value since the pairwise comparison matrix exists at each level, then local priorities must be synthesized to obtain global priorities. Procedures for synthesizing differ according to the shape of the hierarchy. The ordering of elements according to relative importance through a synthesis procedure is called priority setting.

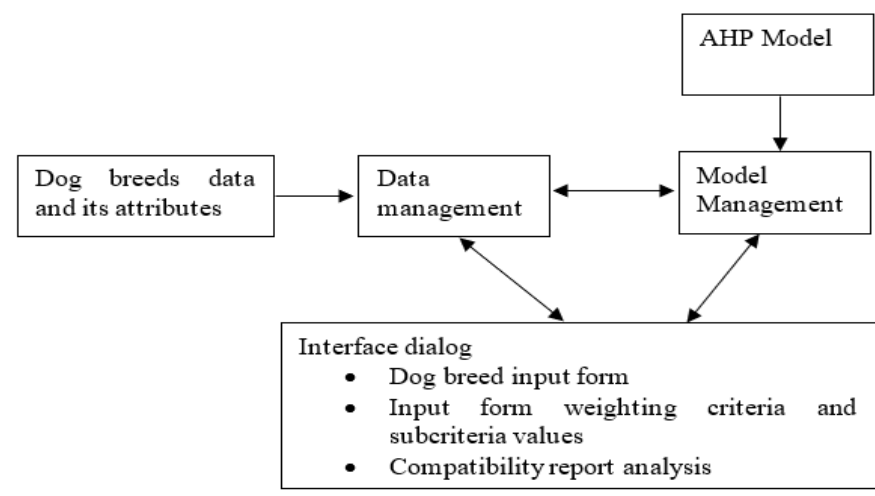

Figure 1: Architecture of DSS for Purebred Dog Selection

\subsection{System Modelling}

The proposed decision support system model consists of several subsystems, the first is a data management subsystem that utilizes structured data sources as a relational database model, the second is a management subsystem model that utilizes the developed analytic models [11,12]. This analytic model would provide to system an analytical capability by utilizing a 
mathematical formula to generate recommendations for the user. Finally, the interface model subsystem is a system component that users used to communicate with the system through an intuitive interface. Figure 1 shows the architectural model of a decision support system for purebred dog selection.

\subsection{Data Analysis and Analytic Model}

Based on the developed analytic model by processing the data sources, several main criteria and sub-criteria have determined in the selection of dog breeds. These criteria and sub-criteria were obtained based on collected and processed data from a primary data source that was the dog kinology information from the FCI and $\mathrm{AKC}$ as the leading world $\operatorname{dog}$ registries to obtain standardization information on physical and psychological characteristics of each breed. The collected data was then compared with secondary data sources obtained from dog lovers communities, dog experts, veterinary and kennel owners to know their experience and preferences in selecting and caring for a purebred dog as a result, eight main criteria and seven sub-criteria were formulated with its degree of intensity used in decision making for the selection of purebred dogs as follows:

1. Intelligence: This criterion defines the ability of the dog to obey commands and its tendency to understand new commands. This criterion has two sub-criteria namely obedience and trainability, which defines how good the dog to obey the commands given by its owner at a given time and how good the dog to understand new commands in minimal repetition count, respectively.

a. Obedience

- High: The dog can obey $70 \%$ or more of the command given every time.

- Medium : The dog can obey $30 \%$ or more of the command given every time.

- Low: The dog can obey less than $30 \%$ of the command given every time.

b. Trainability

- High: The dog can understand new command with training frequency less than 16 reps.

- Medium: The dog can understand new command with traning frequency 16 to 40 reps.

- Low: The dog can understand new command with training frequency more than 40 reps.

2. Hygiene: This Criterion defines the level of frequency of a dog to produce natural waste that beyond its control like feather loss (shedding) and drooling. This criterion has two subcriteria namely non-shedding and non-drooling.

a. Non-Shedding

- High: Feather loss rarely occurs.

- Medium: Feather loss occurs periodically/seasonally naturally.

- Low: Feather loss occurs most of the time naturally

b. Non-Drooling

- High: Drooling never or rarely happen..

www.astesj.com
- Medium: Drooling sometimes occur in certain condition..

- Low: Drooling occurs frequently.

3. Maintenance: This Criterion determines how necessary a specific dog according to the breed type requires special care outside of its daily care, the specific treatment like advanced grooming, specific nail care, and any other special treatment. The intensity degree for this criterion are:

- High: The dog do not need or only require minimal additional care like simple grooming

- Medium : The dog need moderate additional care like full grooming

- Low: The dog needs special care since that affects its hygiene and healthy body. the dog might need advanced grooming, nail trimming, and any other services.

4. Friendliness: This Criterion defines the aggressiveness level of a purebred dog naturally shown against the subject (humans and other animals). Excessive barking toward unfamiliar subject indicates mild aggressive behavior, and severe aggressive behavior has indicated by a tendency to attack the subjects. The intensity degree for this criterion are:

- High: The dog is friendly and generally does not bark if there is no provocative action from the subject.

- Medium: The dog might always bark at any unfamiliar subject.

- Low: The dog has tendency to engage in aggresive behavior including barking and attacking unfamiliar subject.

5. Adaptability: This Criterion defines the level of adaptability of dog breed toward human, other pet, and environment, good adaptability allows the dog to adjust well with the environment and made a good relationship with the adopter and other pets.

a. Adaptation to Human

- High: The dog can adapt well and easy to make new connection with human.

- Medium : The dog can still adapt well but need time to make a new relationship with a human.

- Low : The dog need much time to adapt and build new relationship with human and sometimes the dog may seem uncomfortable until they fully adapt with the human counterparts

b. Adaptation to other pets

- High: The dog highly adaptable and can live along with other pets without problems.

- Medium: The dog can adapt well with a certain pet usually with same species but can behave aggressively to the other one.

- Low: The dog does not like other animal and has a tendency to consider other dog as a rival.

c. Adaptation to environment

- High: The dog highly adaptable to various environmental conditions and has a high tolerance with weather changes. 
- Medium: The dog can still adapt well with certain enviromental condition but sometimes need human to help the adaptability process.

- Low: The dog needs a certain environmental condition to live well and very dependant to human to help the adaptability process.

6. Popularity: This criterion defines how popular a breed based on the rankings released by AKC. AKC annually updates the most popular dog ranking data based on genealogy registration, contests, and surveys. For adopters to have a popular dog has benefits such as the ease of joining a large community of dog owners. The intensity degree for this criterion are:

- High: Very popular dog breed, which is a breed that ranks between 1 to 25 according to the latest AKC annual List.

- Medium: moderately popular dog breed, which is a breed that ranks between 26 to 100 according to the latest AKC annual List.

- Low: Less popular dog breed, which is a breed that ranks below 100 according to the latest AKC annual List.

7. Longevity: The life expectancy of a dog breed. According to research, each dog has a lifespan between 5 to 15 years depending on the breed [13]. The intensity degree for this criterion are:

- High: The dog breed with an average lifespan above 12 years.

- Medium: The dog breed with an average lifespan between 9 to 12 years

- Low: The dog breed with an average lifespan below 9 years.

8. Impression: Adopters based criteria that express the level of their impression on the certain dog breed subjectively (usually about the dog physically attraction). The intensity degree for this criterion are:

- High: The prospective adopter has a great impression of the breed type.

- Medium: The prospective adopter has a good impression of the breed type.

- Low: The prospective adopter is less interested in breed type.

\section{Result and Discussion}

\subsection{System Design and Evaluation}

Based on the designed model, the data of all breeds that have been officially recognized by FCI and AKC were 193 breeds and have been recorded in the database. Table 1 shows some of the breeds available in the system.

Table 1: List of some dog breeds

\begin{tabular}{|l|l|}
\hline 001 & Chihuahua \\
\hline 002 & Pekingese \\
\hline 003 & Pomeranian \\
\hline 004 & Golden Retriever \\
\hline 005 & Maltese \\
\hline 006 & German Shepherd \\
\hline 007 & Border Collie \\
\hline 008 & Shih Tzu \\
\hline
\end{tabular}

\begin{tabular}{|l|l|}
\hline 009 & Labrador Retriever \\
\hline 010 & Chow-chow \\
\hline 011 & Doberman Pinscher \\
\hline 012 & Bulldog \\
\hline 013 & Saint Bernard \\
\hline 014 & Boxer \\
\hline 015 & Bloodhound \\
\hline 016 & Shiba Inu \\
\hline 017 & Poodle (Toy) \\
\hline 018 & Dalmatian \\
\hline
\end{tabular}

Then from the designed analytical model, each data criteria, sub-criteria, and intensity levels have been structured into the database as shown in table 2 to table 4, there were eight main criteria, which three of them have sub-criteria, finally, there were three degrees of intensity data that will be used for each criterion and sub-criterion.

Table 2: List of Criteria

\begin{tabular}{|l|l|}
\hline ID Criteria & Criteria \\
\hline 1 & Intelligence \\
\hline 2 & Hygiene \\
\hline 3 & Maintenance \\
\hline 4 & Friendliness \\
\hline 5 & Adaptability \\
\hline 6 & Popularity \\
\hline 7 & Longevity \\
\hline 8 & Impression \\
\hline
\end{tabular}

Table 3: List of SubCriteria

\begin{tabular}{|l|l|l|}
\hline ID SubCriteria & Criteria & Subcriteria \\
\hline 1 & Intelligence & Obedience \\
\hline 2 & Hygiene & Trainability \\
\hline 1 & Maintenance & Non-Shedding \\
\hline 2 & Friendliness & Non-Drolling \\
\hline 1 & Adaptability & Human \\
\hline 2 & Popularity & Animal \\
\hline 3 & Longevity & Environment \\
\hline
\end{tabular}

Table 4: List of Intensity

\begin{tabular}{|l|l|}
\hline ID Intensity & Intensity \\
\hline 1 & Low \\
\hline 2 & Medium \\
\hline 3 & High \\
\hline
\end{tabular}

The system provided several preset (pre-configured set) options that can be used by the user as a prospective adopter for finding the right breed, Besides that users can manually configure the settings tailored to their needs. The preset provided by the system refers to collected data from the FCI and AKC knowledge base in their official website (http://fci.be) and (www.akc.org) plus collaboration with empirical data obtained from the dog lovers community, dog experts, and veterinary. Besides, each preset available will also take the subjective preferences of the user into account by giving the impression criterion certain weight value that will be used in computing to generate recommendations.

There were several pre-configured sets provided by the system that can be used by users in the selection of dog breeds. One 
example of a preset provided by the system was namely workingdog preset. This preset was useful for finding dog breed recommendations that have the potential to support humans jobs. This preset has intended to provide recommendations to prospective adopters who need breed types that have great potential to be trained for specific works according to the needs of prospective adopters, for example, dogs that are planned to work as herding dogs for livestock, these dogs must meet the requirement needed as easy to train, independent, easily adaptable, and have good territorial awareness [14]. Another pre-configure set provided by the system wasthe Family-dog preset to help the user to find a dog who can be a great companion for its adopter. The dog expected to be friendly with all family members including children and to other pets such as cats and rabbits. The dogs that are suitable for this role were the dog whose main character is friendly, physically attractive, and have great adaptability to humans and other pets. Both preset certainly have different weighting values and criterion importance ranking, Working-dog preset has higher weighting values for intelligence and adaptability criteria (table 5), while family-dog presets prioritize impression criteria since it is primarily used by an adopter who chooses a dog as a family companion as same as the friendliness criteria which is the second most important criterion for this preset category (table 6). From the criteria weighting matrix, we can get a rank of each criterion based on the eigenvector value. Table 7 and table 8 shows the criteria ranking for working-dog presets and the criteria ranking for family-dog presets respectively.

Table 5: Weighting value matriks for working-dog preset

\begin{tabular}{|l|l|l|l|l|l|l|l|l|}
\hline & Intelligence & Hygiene & Maintenance & Friendliness & Adaptability & Popularity & Longevity & Impression \\
\hline Intelligence & 1 & 7 & 4 & 9 & 2 & 5 & 8 & 6 \\
\hline Hygiene & 0.14 & 1 & 0.33 & 2 & 0.22 & 0.5 & 2 & 0.5 \\
\hline Maintenance & 0.25 & 3 & 1 & 5 & 0.33 & 2 & 4 & 3 \\
\hline Friendliness & 0.11 & 0.5 & 0.2 & 1 & 0.14 & 0.25 & 0.5 & 0.33 \\
\hline Adaptability & 0.5 & 5 & 3 & 7 & 1 & 3 & 6 & 4 \\
\hline Popularity & 0.2 & 2 & 0.5 & 4 & 0.33 & 1 & 3 & 2 \\
\hline Longevity & 0.13 & 0.5 & 0.25 & 2 & 0.16 & 0.33 & 1 & 0.5 \\
\hline Impression & 0.16 & 2 & 0.33 & 3 & 0.25 & 0.5 & 2 & 1 \\
\hline Total & 2.49 & 21 & 9.61 & 33 & 4.41 & 12.58 & 26.5 & 17.3 \\
\hline
\end{tabular}

Table 6: Weighting value matriks for family-dog preset

\begin{tabular}{|l|l|l|l|l|l|l|l|l|}
\hline & Intelligence & Hygien & Maintenance & Friendliness & Adaptability & Popularity & Longevity & Impression \\
\hline Intelligence & 1 & 0.2 & 4 & 0.25 & 3 & 0.5 & 2 & 0.16 \\
\hline Hygiene & 5 & 1 & 8 & 2 & 7 & 4 & 6 & 0.5 \\
\hline Maintenance & 0.25 & 0.125 & 1 & 0.14 & 0.5 & 0.25 & 0.33 & 0.11 \\
\hline Friendliness & 4 & 0.5 & 7 & 1 & 6 & 3 & 5 & 0.33 \\
\hline Adaptability & 0.33 & 0.14 & 2 & 0.16 & 1 & 0.25 & 0.5 & 0.125 \\
\hline Popularity & 2 & 0.25 & 4 & 0.33 & 4 & 1 & 3 & 0.2 \\
\hline Longevity & 0.5 & 0.16 & 3 & 0.2 & 2 & 0.33 & 1 & 0.14 \\
\hline Impression & 6 & 2 & 9 & 3 & 8 & 5 & 7 & 1 \\
\hline Total & 14.8 & 4.38 & 38 & 7.08 & 31.5 & 14.33 & 24.83 & 2.56 \\
\hline
\end{tabular}

Table 7: Criterion importance rank for working-dog preset

\begin{tabular}{|l|l|l|}
\hline Criteria & Eigenvector value & Priority Rank \\
\hline Intelligence & 0.3862 & 1 \\
\hline Adaptability & 0.2407 & 2 \\
\hline Maintenance & 0.1329 & 3 \\
\hline Popularity & 0.0892 & 4 \\
\hline
\end{tabular}

$\underline{\text { www.astesj.com }}$

\begin{tabular}{|l|l|l|}
\hline Impression & 0.0623 & 5 \\
\hline Hygiene & 0.0489 & 6 \\
\hline Longevity & 0.0342 & 7 \\
\hline Friendliness & 0.0236 & 8 \\
\hline & Consistency ratio & $2.63 \%$ \\
\hline
\end{tabular}

Table 8: Criterion importance rank for family-dog preset

\begin{tabular}{|l|l|l|}
\hline Criteria & Eigenvector value & Priority Rank \\
\hline Intelligence & 0.3433 & 1 \\
\hline Adaptability & 0.246 & 2 \\
\hline Maintenance & 0.1762 & 3 \\
\hline Popularity & 0.0872 & 4 \\
\hline Impression & 0.0597 & 5 \\
\hline Hygiene & 0.0399 & 6 \\
\hline Longevity & 0.0272 & 7 \\
\hline Friendliness & 0.0204 & 8 \\
\hline & Consistency ratio & $3.69 \%$ \\
\hline
\end{tabular}

The next step after all the criteria and sub-criteria weights have obtained from the preceding process was to perform the computation on all of these weights value to each official breed stored in the database to get the compatibility score of each dog breed. The process of calculating the compatibility value of each alternative has done by weighting the intensity of each criterion and sub-criterion to each dog breed profile based on information stored in the data source. In this example, the subjective preference of prospective adopter was ignored by weighting the intensity of the impression criteria for each breed using the same value. The compatibility result of each breed for Working-dog presets and Family-dog presets were shown in Figure 2 and Figure 3, respectively.

\section{ALTERNATIVE COMPATIBILITY SCORE}

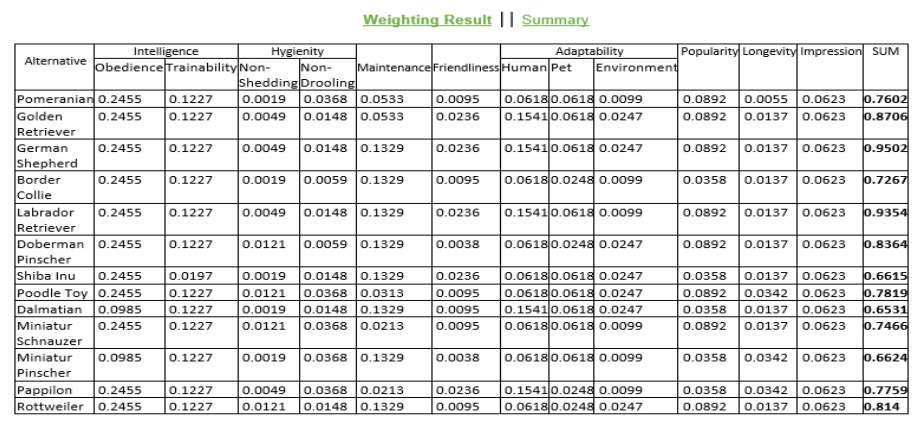

Figure 2: Breed compatibility result for working-dog preset

\section{ALTERNATIVE COMPATIBILITY SCORE}

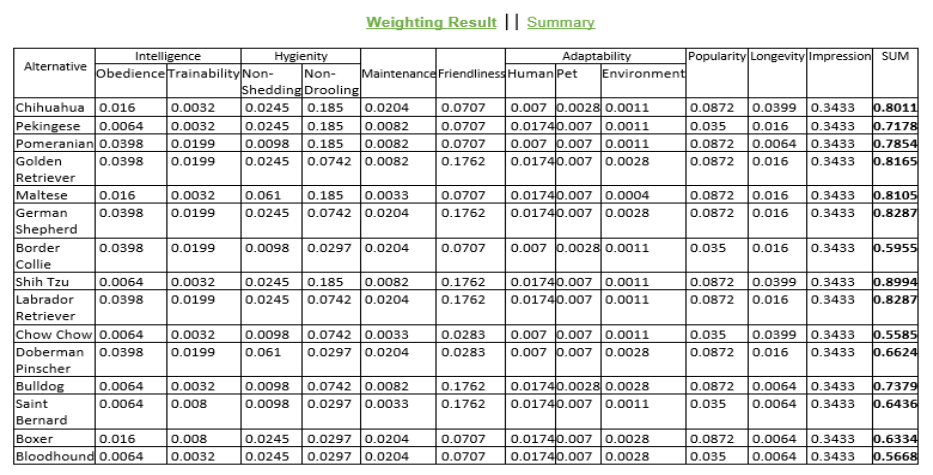

Figure 3: Breed compatibility result for family-dog preset 
Based on the results of the compatibility value, the system will display three dog breeds by the highest compatibility value to be used as recommendations for prospective adopters. Figure 4 and Figure 5 shows the three recommended dog breed by the system for working dog presets and family dog presets respectively.

\section{Working Dog Recommendations}

\begin{tabular}{|l|l|l|}
\hline Picture & \multicolumn{1}{|c|}{ Dog Breed } & Total Score \\
\hline & Gernan shepherd & 0.9502 \\
\hline & Labrador retriever & 0.9354 \\
\hline & Golden retriever & 0.8706 \\
\hline
\end{tabular}

Figure 4: Working-dog recommendation by system

\section{Family Dog Recommendations}

\begin{tabular}{|c|l|l|}
\hline Picture & \multicolumn{1}{|c|}{ Dog Breed } & \multicolumn{1}{|c|}{ Total Score } \\
\hline & Slih Tzu & 0.8994 \\
\hline & Papillon & 0.8882 \\
\hline & Siberian Husky & 0.8752 \\
\hline
\end{tabular}

Figure 5: Family-dog recommendation by system

\section{Conclusion}

Based on research conducted, researchers have designed an analytical model that uses multiple data sources to gather information regarding the physical and psychological characteristics of each dog breed for quantitative analysis without rule out the personal subjectivity of prospective dog adopters. The result was a decision support system that can assist prospective dog adopters in choosing the breed that suits their needs and preferences best by making recommendations based on the dog breed compatibility ranking towards their preference. The final decision remains the prerogative of prospective adopters with all the consequences.

\section{Conflict of Interest}

The authors declare no conflict of interest.

\section{References}

[1] P. Chyan, "Decision Support System for Selection of Dog Breeds," in 2018 International Seminar on Research of Information Technology and Intelligent Systems (ISRITI), 343-346, 2018, doi:10.1109/ISRITI.2018.8864327.

[2] S. Barnard, C. Passalacqua, A. Capra, S. Marshall-Pescini, E.P. Previde, P. Valsecchi, "Social behavioral profile of different dog breeds," Journal of Veterinary Behavior, $\quad \mathbf{6}(1), \quad 83-84, \quad 2011$, doi:https://doi.org/10.1016/j.jveb.2010.09.004.

[3] M. Nitzschner, A.P. Melis, J. Kaminski, M. Tomasello, "Dogs (Canis familiaris) evaluate humans on the basis of direct experiences only," PloS One, 7(10), e46880-e46880, 2012, doi:10.1371/journal.pone.0046880.

[4] S. Coren, The Intelligence of Dogs: A Guide to Thoughts, Emotions, and Inner Lives of Our Canine Companions, Atria Books, 2006.

[5] S. Coren, How Dogs Think, First Free Press, Simon \& Schuster, New York, 2004.

[6] A. Miklosi, Dog Behaviour, Evolution, and Cognition, 2nd Editio, Oxford University Press, 2016.

[7] A.R. Michell, "Longevit of British breeds of dog and its relationships withsex, size, cardiovascular variables and disease," Veterinary Record, 145(22),
625-629, 1999, doi:10.1136/vr.145.22.625.

[8] J. Anderson, M. Krieger, M. Safley, A comparative analysis of alpaca breed type and standards, Northwest Alpaca at Wayback Machine, 2009.

[9] C. Coile, Encyclopedia of Dog Breeds, Barron's Educational series, 2015.

[10] T.. Saaty, Fundamentals of Decision Making and Priority Theory with the Analytic Hierarchy Process, RWS Publication, 2000.

[11] P. Chyan, "Design of intelligent camera-based security system with image enhancement support," Journal of Physics: Conference Series, 1341, 42009, 2019, doi:10.1088/1742-6596/1341/4/042009.

[12] P. Chyan, "Image Enhancement Based On Bee Colony Algorithm," Journal of Engineering and Applied Sciences, 14(1), 43-49, 2019.

[13] E. Korec, O. Chalupa, M. Hančl, J. Korcová, M. Bydžovská, "Longevity of Cane Corso Italiano dog breed and its relationship with hair colour," Open Veterinary Journal, 7(2), 170-173, 2017, doi:10.4314/ovj.v7i2.15.

[14] M.J. Dotson, E.M. Hyatt, "Understanding dog-human companionship," Journal of Business Research, 61(5), 457-466, 2008, doi:https://doi.org/10.1016/j.jbusres.2007.07.019. 